\title{
Overlapping Nonmatching Grid Method for the Ergodic Control Quasi Variational Inequalities
}

\author{
H. Mécheri, S. Saadi \\ Department of Mathematics, Badji Mokhtar University, Annaba, Algeria \\ Email: halima.mecheri51@gmail.com, saadisamira69@yahoo.fr
}

Received February 16, 2013; revised March 17, 2013; accepted March 31, 2013

Copyright (C) 2013 H. Mécheri, S. Saadi. This is an open access article distributed under the Creative Commons Attribution License, which permits unrestricted use, distribution, and reproduction in any medium, provided the original work is properly cited.

\begin{abstract}
In this paper, we provide a maximum norm analysis of an overlapping Schwarz method on nonmatching grids for a quasi-variational inequalities related to ergodic control problems studied by M. Boulbrachène [1], where the "discount factor" (i.e., the zero order term) is set to 0, we use an overlapping Schwarz method on nonmatching grid which consists in decomposing the domain in two sub domains, where the discrete alternating Schwarz sequences in sub domains converge to the solution of the ergodic control IQV for the zero order term. For $\alpha \in] 0,1[$ and under a discrete maximum principle we show that the discretization on each sub domain converges quasi-optimally in the $L^{\infty}$ norm to 0 .
\end{abstract}

Keywords: Quasi Variational Inequalities; Ergodic Control; Schwarz Method; Finite Element Method

\section{Introduction}

The Schwarz alternating method can be used to solve elliptic boundary value problems on domains which consist of two or more overlapping sub domains.

The solution is approximated by an infinite sequence of functions which results from solving a sequence of elliptic boundary value problems in each of the sub domain.

In this paper, we are interested in the error analysis in the maximum norm for the obstacle problem in the context of overlapping nonmatching grids: we consider a domain $\Omega$ which is the union of two overlapping subdoains where each sub domain has its own triangulation. This kind of discretizations is very interesting as they can be applied to solving many practical problems which cannot be handled by global discretizations. They are earning particular attention of computational experts and engineers as they allow the choice of different mesh sizes and different orders of approximate polynomials in different sub domains according to the different properties of the solution and different requirements of the practical problems.

We study a new approach for the finite element approximation for the ergodic problem where the obstacle is related to a solution. We consider a domain which the union of two overlapping sub-domains where each sub domain has its own generated triangulation. The grid points on the sub-domain boundaries need not much the grid points from the other sub-domains. Under a discrete maximum principle, we show that the discretization on each sub-domain converges quasi-optimally in the $L^{\infty}$ norm.

In the first section we study the Schwarz method for the ergodic control Quasi-variational inequalities; we state the continuous alternating Schwarz sequence for quasivariational inequalities, and define their respective finite element counterparts in the context of overlapping grids.

In Section 2, we give a simple proof for the main result concerning error estimates in the $L^{\infty}$ norm for the problem studied, taking into account the combination of Jinping Zeng \& Shuzi Zhou [2] geometrical convergence and P. L. Lions, B. Perthame [3] quasi-variational inequalities and ergodic impulse control.

\section{Schwarz Method for the Ergodic Control Quasi-Variational Inequalities}

We begin by down a classical results related to ergodic control quasi-variational inequalities [1-18].

It is well known that impulse control problems for reflected diffusion process may be solved by considering the solution of quasi variational inequalities (QVI) (see Bensoussan [4], A. Bensoussan and J. L. Lions [5]). A typical example is the following: 


$$
\left\{\begin{array}{l}
a\left(u_{\alpha}, v-u_{\alpha}\right)+\left(u_{\alpha}, v-u_{\alpha}\right) \geq\left(f, v-u_{\alpha}\right) \\
u_{\alpha} \in H^{1}(\Omega), u_{\alpha} \leq M u_{\alpha} ; v \in H^{1}(\Omega), v \leq M u_{\alpha}
\end{array}\right.
$$

where $\Omega$ is given bounded smooth open set in $\square^{N}$, $\alpha>0, f$ is given function, the cost function $M u$ represents the obstacle of impulse control defined by:

$$
\begin{aligned}
& M \varphi(x)=k+\inf (x+\xi) \\
& \xi \geq 0, x+\xi \in \Omega, \text { where } k>0
\end{aligned}
$$

where $k$ is a positive number, $M$ is an operator defined on $C(\bar{\Omega})$ and assumed to map into itself, that is,

$$
M \varphi(x) \leq M \tilde{\varphi}(x) \text { whenever } \varphi(x) \leq \tilde{\varphi}(x)
$$

$a(u, v)=\int_{\Omega} \nabla u \cdot \nabla v \mathrm{~d} x ;$ and $(.,$.$) denotes the inner product$ on $\Omega$.

It has been proved that the long run average cost for this problem solves the ergodic QVI. More precisely, denoting by:

$$
\langle w\rangle=\frac{1}{\Omega} \int_{\Omega} w \mathrm{~d} x, w_{\alpha}=u_{\alpha}-\left\langle u_{\alpha}\right\rangle \text { and } \lambda_{\alpha}=\alpha\left\langle u_{\alpha}\right\rangle .
$$

P. L. Lions and B. Perthame [3] proved that the solution $\left(w_{\alpha}, \lambda_{\alpha}\right)$ of the QVI :

$\left\{\begin{array}{l}a\left(w_{\alpha}, v-w_{\alpha}\right)+\left(w_{\alpha}, v-w_{\alpha}\right) \geq\left(f-\lambda_{\alpha}, v-w_{\alpha}\right) \\ w_{\alpha} \in H^{1}(\Omega), w_{\alpha} \leq M w_{\alpha} ; v \in H^{1}(\Omega), v \leq M w_{\alpha},\left\langle w_{\alpha}\right\rangle=0\end{array}\right.$

converges to the solution of the ergodic control QVI :

$$
\left\{\begin{array}{l}
a\left(w_{0}, v-w_{0}\right)+\left(w_{0}, v-w_{0}\right) \geq\left(f-\lambda_{0}, v-w_{0}\right) \\
w_{0} \in H^{1}(\Omega), w_{0} \leq M w_{0} ; v \in H^{1}(\Omega), v \leq M w_{0},\left\langle w_{0}\right\rangle=0
\end{array}\right.
$$

As stated in the following theorem.

Theorem 1 [1]: As $\alpha$ goes to $0^{+}, \lambda_{\alpha}$ converges uniformly in $C(\bar{\Omega})$ to some constant $\lambda_{0}$, and $w_{\alpha}$ converges uniformly in $C(\bar{\Omega})$ and strongly in $H^{1}(\Omega)$ to $w_{0}$. Moreover $\left(\lambda_{0}, w_{0}\right)$ is the unique solution of the quasi variational inequality of the ergodic control prob$\operatorname{lem}(5)$.

\section{Problem Position}

Let $\alpha$ be fixed in the open interval $] 0,1[$ and set. Then, one can easily see that problem (1) is equivalent to the following QVI:

$$
\left\{\begin{array}{l}
b\left(u_{\alpha}, v-u_{\alpha}\right) \geq\left(f+\gamma u_{\alpha}, v-u_{\alpha}\right) \\
u_{\alpha} \in H^{1}(\Omega), u_{\alpha} \leq M u_{\alpha} \\
v \in H^{1}(\Omega), v \leq M u_{\alpha}
\end{array}\right.
$$

where

$$
b(u, v)=a(u, v)+(u, v), \text { and } \gamma=1-\alpha
$$

Thanks to [5], (1) or (6) has a unique solution. Also, notice that, as the bilinear form (7) is independent of $\alpha$, the left hand side of (6) is independent of $\alpha$ too.

\section{The Schwarz Method for the Obstacle}

We decompose $\Omega$ into two overlapping polygonal sub domains $\Omega_{1}$ and $\Omega_{2}$, such that

$$
\Omega=\Omega_{1} \cup \Omega_{2}
$$

and $u_{\alpha}$ satisfies the local regularity condition

$$
u_{\alpha} / \Omega_{i} \in W^{2, p}\left(\Omega_{i}\right), 2 \leq p<\infty
$$

We denote by $\partial \Omega_{i}$ the boundary condition of $\Omega_{i}$ and $\Gamma_{i}=\partial \Omega_{i} \cap \Omega_{i}$ the intersection of $\bar{\Gamma}_{i}$ and $\bar{\Gamma}_{j}$ is assumed to be empty.

\subsection{The Schwarz Sequences for Problem (4)}

We denote by:

$$
w_{\alpha}^{n+1}=u_{\alpha}^{n+1}-\left\langle u_{\alpha}^{n+1}\right\rangle \text { and } \lambda_{\alpha}^{n+1}=\alpha^{n+1}\left\langle u_{\alpha}^{n+1}\right\rangle, n \geq 0
$$

Choosing $w_{\alpha}^{0}=w_{0}$ such that $w_{0}$ the unique solution of:

$$
\left\{\begin{array}{l}
a\left(w_{0}, v-w_{0}\right)+\left(w_{0}, v-w_{0}\right) \geq\left(f-\lambda_{0}, v-w_{0}\right) \\
w_{0} \in H^{1}(\Omega), w_{0} \leq M w_{0} \\
v \in H^{1}(\Omega), v \leq M w_{0},\left\langle w_{0}\right\rangle=0
\end{array}\right.
$$

We respectively define $w_{\alpha i}^{n+1}$ the alternating Schwarz sequences on $\Omega_{i}$ such that:

$$
\left\{\begin{array}{l}
a\left(w_{\alpha i}^{n+1}, v-w_{\alpha i}^{n+1}\right)+\left(w_{\alpha i}^{n+1}, v-w_{\alpha i}^{n+1}\right) \geq\left(f_{i}-\lambda_{\alpha i}^{n+1}, v-w_{\alpha i}^{n+1}\right) \\
w_{\alpha i}^{n+1}=w_{\alpha i}^{n} \text { on } \Gamma_{i}, w_{\alpha i}^{n+1} \leq M w_{\alpha i}^{n} \\
v \in H^{1}(\Omega), v \leq M w_{\alpha i}^{n},\left\langle w_{\alpha i}^{n+1}\right\rangle=0
\end{array}\right.
$$

where $i=1,2$ and $f_{i}=f / \Omega_{i}$.

\subsection{The Continuous Schwarz Sequences for Principal Problem}

We consider the following problem:

$$
\left\{\begin{array}{l}
b\left(u_{\alpha}, v-u_{\alpha}\right) \geq\left(f+\gamma u_{\alpha}, v-u_{\alpha}\right) \\
u_{\alpha} \in H^{1}(\Omega), u_{\alpha} \leq M u_{\alpha} ; v \in H^{1}(\Omega), v \leq M u_{\alpha}
\end{array}\right.
$$

Choosing $u_{\alpha}^{0}=u_{0}$, solution of:

$$
\left\{\begin{array}{l}
a\left(u_{0}, v-u_{0}\right) \geq\left(f, v-u_{0}\right) \\
u_{0} \in H^{1}(\Omega), u_{0} \leq M u_{0} \\
v \in H^{1}(\Omega), v \leq M u_{0}
\end{array}\right.
$$

We respectively define the alternating Schwarz sequences $\left(u_{\alpha 1}^{n+1}\right)$ on $\Omega_{1}$ such that: $\Omega=\Omega_{1} \cup \Omega_{2}$ and; 


$$
\begin{gathered}
\left\{\begin{array}{l}
b\left(u_{\alpha 1}^{n+1}, v-u_{\alpha 1}^{n+1}\right) \geq\left(f+\gamma u_{\alpha 1}^{n+1}, v-u_{\alpha 1}^{n+1}\right), \forall v \in H^{1}(\Omega) \\
u_{\alpha 1}^{n+1} \leq M u_{\alpha 1}^{n}, M u_{\alpha 1}^{n}>0 \\
u_{\alpha 1}^{n+1}=u_{\alpha 2}^{n} \text { on } \Gamma_{1}, v=u_{\alpha 2}^{n} \text { on } \Gamma_{1}
\end{array}\right. \\
\left\{\begin{array}{l}
b\left(u_{\alpha 2}^{n+1}, v-u_{\alpha 2}^{n+1}\right) \geq\left(f+\gamma u_{\alpha 2}^{n+1}, v-u_{\alpha 2}^{n+1}\right), \forall v \in H^{1}(\Omega) \\
u_{\alpha 2}^{n+1} \leq M u_{\alpha 2}^{n}, M u_{\alpha 2}^{n}>0 \\
u_{\alpha 2}^{n+1}=u_{\alpha 1}^{n+1} \text { on } \Gamma_{2}, v=u_{\alpha 1}^{n+1} \text { on } \Gamma_{2} \\
\quad b_{i}\left(u_{\alpha}, v\right) \\
=a\left(u_{\alpha}, v\right)+\gamma \int_{\Omega_{i}} u_{\alpha} \cdot v \mathrm{~d} x, i=1,2
\end{array}\right.
\end{gathered}
$$

Lemma 1 [3]: for each $n \geq 0$;

$$
M u_{\alpha 1}^{n+1}\left(\operatorname{respec} M u_{\alpha 2}^{n+1}\right) \in C(\bar{\Omega})
$$

\section{The Discret Problem}

We suppose for simplicity that $\Omega$ is polyhedral. Let $r_{h}$ be a regular, quasi uniform triangulation of $\Omega$ into nsimplexes of diameter less than $h$.

We denote by $V_{h}$ the standard piecewise linear finite element espace, we consider the discrete variational inequality:

$$
\left\{\begin{array}{l}
a\left(u_{\alpha h}, v_{h}-u_{\alpha h}\right)+\left(u_{\alpha h}, v_{h}-u_{\alpha h}\right) \geq\left(f, v_{h}-u_{\alpha h}\right) \\
u_{\alpha h} \in V_{h}, u_{\alpha h} \leq r_{h} M u_{\alpha h} ; v_{h} \in V_{h}, v_{h} \leq r_{h} M u_{\alpha h}
\end{array}\right.
$$

Thanks to ([6]), QVI (18) has a unique solution.

\subsection{The Discrete Maximum Principle [7]}

We assume that the matrix A with generic coefficient is a M-matrix.

$$
B_{i j}\left(\varphi_{i}, \varphi_{j}\right), 1 \leq i, j \leq m(h)
$$

As for the continuous problem, it is easy to see that $u_{\alpha h}$, the solution of (18), is also solution to the following (QVI):

$$
\left\{\begin{array}{l}
b\left(u_{\alpha h}, v_{h}-u_{\alpha h}\right) \geq\left(f+\gamma u_{\alpha h}, v_{h}-u_{\alpha h}\right) \\
u_{\alpha h} \in V_{h}, u_{\alpha h} \leq r_{h} M u_{\alpha h} ; v_{h} \in V_{h}, v_{h} \leq r_{h} M u_{\alpha h}
\end{array}\right.
$$

Theorem 2 [1]: Let $u_{\alpha}$ and $u_{\alpha h}$ (the discrete solution). Then, there exists a constant independent of $\alpha$ the both and $h$ such that

$$
\left\|u_{\alpha}-u_{\alpha h}\right\|_{\infty} \leq C \cdot \alpha^{-2} h^{2}|\log h|^{3}
$$

Theorem 3 [1]: Under conditions of theorem 1 and 2, we have

$$
\begin{aligned}
& \lim _{h \rightarrow 0}|| w_{\alpha h}-w_{0} \|_{\infty}=0 \\
& \lim _{h \rightarrow 0}\left|\lambda_{\alpha h}-\lambda_{0}\right|=0
\end{aligned}
$$

Note that $w_{\alpha h}$ and $\lambda_{\alpha h}$ is the finite element approximation of $w_{\alpha}$ and $\lambda_{\alpha}$ respectively.

\subsection{The Discrete Schwarz Sequences}

Let $V_{h i}=V_{h}\left(\Omega_{i}\right)$ be the space of continuous piecewise linear function on $\tau_{h i}$ which vanish on $\partial \Omega \bigcap \partial \Omega_{i}$.

For $w \in C\left(\Gamma_{i}\right)$, we define

$$
\begin{aligned}
& V_{h i}^{(w)}= \\
& \left\{v \in V_{h} / v=0 \text { on } \partial \Omega \bigcap \partial \Omega_{i}, v=\pi_{h i}(w) \text { on } \Gamma_{i}\right\}
\end{aligned}
$$

where $\pi_{h i}$ denotes the interpolation operator on $\Gamma_{i}$.

For $i=1,2$. Let $\tau_{h i}$ be a standard regular finite element triangulation in $\Omega_{i}, h_{i}$ being the mesh size.

We suppose that the two triangulations are mutually independent on $\Omega_{1} \cup \Omega_{2}$ a triangle belonging to one triangulation does not necessarily belong to the other.

Choosing $u_{\alpha h}^{0}=u_{0 h}$, such that $u_{0 h}$ is a solution of the following inequation:

$$
\left\{\begin{array}{l}
a\left(u_{0 h}, v_{h}-u_{0 h}\right) \geq\left(f, v_{h}-u_{0 h}\right) \\
u_{0 h} \in V_{h}, u_{0 h} \leq r_{h} M u_{0 h} ; v_{h} \in V_{h}, v_{h} \leq r_{h} M u_{0 h}
\end{array}\right.
$$

We define the alternating Schwarz sequences $\left(u_{\alpha 1 h}^{n+1}\right)$ on $\Omega_{1}$ such that:

$$
\left\{\begin{array}{l}
b_{1}\left(u_{\alpha 1 h}^{n+1}, v_{h}-u_{\alpha 1 h}^{n+1}\right) \geq\left(f_{1}+\gamma u_{\alpha 1 h}^{n+1}, v_{h}-u_{\alpha 1 h}^{n+1}\right), v_{h} \in V_{h} \\
u_{\alpha 1 h}^{n+1} \leq r_{h} M u_{\alpha 1 h}^{n}, M u_{\alpha 1 h}^{n}>0 \\
u_{\alpha 1 h}^{n+1}=u_{\alpha 2 h}^{n} \text { on } \Gamma_{1}, v_{h}=u_{\alpha 2 h}^{n} \text { on } \Gamma_{1}
\end{array}\right.
$$

And $\left(u_{\alpha 2 h}^{n+1}\right)$ on $\Omega_{2}$ such that:

$$
\left\{\begin{array}{l}
b_{2}\left(u_{\alpha 2 h}^{n+1}, v_{h}-u_{\alpha 2 h}^{n+1}\right) \geq\left(f_{2}+\gamma u_{\alpha 2 h}^{n+1}, v_{h}-u_{\alpha 2 h}^{n+1}\right), \\
v_{h} \in V_{h}, u_{\alpha 2 h}^{n+1} \leq r_{h} M u_{\alpha 2 h}^{n}, M u_{\alpha 2 h}^{n}>0 \\
u_{\alpha 1 h}^{n+1}=u_{\alpha 1 h}^{n+1} \text { on } \Gamma_{2}, v_{h}=u_{\alpha 1 h}^{n+1} \text { on } \Gamma_{2}
\end{array}\right.
$$

Notation: We will adapt the following notations:

$$
\begin{aligned}
& \|\|_{i}=\|\cdot\|_{L^{\infty}\left(\Gamma_{i}\right)}, i=1,2 \\
& \|\cdot\|_{i}=\|\cdot\|_{L^{\infty}\left(\Omega_{i}\right)}, i=1,2
\end{aligned}
$$

\section{5. $L^{\infty}$-Error Analysis}

Lemma 2 [8]: $\quad B=\left(b_{i j}\right)_{i, j=\{1, \cdots, N\}}$ is M-matrix such that

$$
\left(b_{i j}\right)=b_{i j}\left(\varphi_{i}, \varphi_{j}\right)
$$

then exists constants $k_{1}, k_{2}$ such that

$$
k_{1}=\sup \left\{w_{h}(x), x \in \eta_{1}\right\} \in(0,1)
$$

and $k_{2}=\sup \left\{w_{h}(x), x \in \eta_{2}\right\} \in(0,1)$ 


$$
\begin{aligned}
& \sup _{\eta_{1}}\left|u_{\alpha 1 h}-u_{\alpha 1 h}^{n+1}\right|_{1} \leq k_{1} \sup _{\eta_{1}}\left|u_{\alpha 1 h}-u_{01 h}^{0}\right|_{1} \\
& \sup _{\eta_{2}}\left|u_{\alpha 2 h}-u_{\alpha 2 h}^{n+1}\right|_{2} \leq k_{2} \sup _{\eta_{2}}\left|u_{\alpha 2 h}-u_{02 h}^{0}\right|_{2}
\end{aligned}
$$

Theorem 4 [8]: $\left(u_{\alpha 1 h}^{n+1}\right),\left(u_{\alpha 2 h}^{n+1}\right) ; n \geq 0$ produced by Schwarz alternating method converges geometrically to $u_{\alpha}$ the solution of obstacle problem, more precisely, there exist $k_{1}, k_{2} \in(0,1)$ which depend only respectively of $\left(\Omega_{1}, \eta_{1}\right)$ and $\left(\Omega_{2}, \eta_{2}\right)$, such that

$$
\begin{aligned}
& \sup _{\bar{\Omega}_{1}}\left|u_{\alpha 1 h}-u_{\alpha 1 h}^{n+1}\right|_{1} \leq k_{1}^{n+1} k_{2}^{n+1} \sup _{\eta_{1}}\left|u_{\alpha 1 h}-u_{01 h}^{0}\right|_{1} \\
& \sup _{\bar{\Omega}_{2}}\left|u_{\alpha 2 h}-u_{\alpha 2 h}^{n+1}\right|_{2} \leq k_{1}^{n+1} k_{2}^{n} \sup _{\eta_{2}}\left|u_{\alpha 2 h}-u_{02 h}^{0}\right|_{2}
\end{aligned}
$$

Theorem 5: Let $h=\max \left(h_{1}, h_{2}\right)$. Then, there exist two constants $\mathrm{C}$ and $k, 0<k<1$, independent of both $h$ and $n$ such that:

$$
\left\|u_{\alpha 1}-u_{\alpha 1 h}^{n+1}\right\|_{\infty} \leq C \cdot \alpha^{-2} h^{2}|\log h|^{4}
$$

and

$$
\left\|u_{\alpha 2}-u_{\alpha 2 h}^{n+1}\right\|_{\infty} \leq C \cdot \alpha^{-2} h^{2}|\log h|^{4}
$$

Proof:

$$
\begin{gathered}
\left\|u_{\alpha 1}-u_{\alpha 1 h}^{n+1}\right\|_{\infty} \\
\leq\left\|u_{\alpha 1}-u_{\alpha 1 h}\right\|_{\infty}+\left\|u_{\alpha 1 h}-u_{\alpha 1 h}^{n+1}\right\|_{\infty} \\
\leq C \cdot \alpha^{-2} h^{2}|\log h|^{3}+k_{1}^{n+1}\left\|u_{\alpha 1 h}-u_{01 h}^{0}\right\|_{1} \\
\left\|u_{\alpha 1}-u_{\alpha 1 h}^{n+1}\right\|_{\infty} \\
\leq C_{1} \cdot \alpha^{-2} h^{2}|\log h|^{3}+k_{1}^{n+1}\left[\left\|u_{\alpha 1}-u_{\alpha 1 h}\right\|_{1}+\left\|u_{\alpha 1}-u_{01 h}^{0}\right\|_{1}\right] \\
\leq C_{1} \cdot \alpha^{-2} h^{2}|\log h|^{3}+k_{1}^{n+1}\left\|u_{\alpha 1}-u_{\alpha 1 h}\right\|_{1}+k_{1}^{n+1}\left\|u_{\alpha 1}-u_{01 h}^{0}\right\|_{1} \\
\leq C_{1} \cdot \alpha^{-2} h^{2}|\log h|^{3}+\left(k_{1}^{n+1}\right) C_{2} \cdot \alpha^{-2} h^{2}|\log h|^{3} .
\end{gathered}
$$

We obtain

$$
\left\|u_{\alpha 1}-u_{\alpha 1 h}^{n+1}\right\|_{\infty} \leq C \cdot \alpha^{-2} h^{2}|\log h|^{4}
$$

The case $i=2$ is similar.

Theorem 6: Let $\left(w_{\alpha i h}^{n+1}\right)$ and $\left(\lambda_{\alpha i h}^{n+1}\right)$, the discretes alternating Schwarz sequences, we have:

$$
\begin{aligned}
& \lim _{h \rightarrow 0}|| w_{\alpha i h}^{n+1}-w_{0 i} \|_{\infty}=0, i=1,2 \\
& \lim _{h \rightarrow 0}\left|\lambda_{\text {aih }}^{n+1}-\lambda_{0 i}\right|=0, i=1,2 .
\end{aligned}
$$

\section{Proof:}

1)

$$
\begin{gathered}
\left\|w_{\alpha 1 h}^{n+1}-w_{01}\right\|_{1} \leq\left\|w_{\alpha 1 h}^{n+1}-w_{\alpha 1 h}\right\|_{1}+\left\|w_{\alpha 1 h}-w_{01}\right\|_{1} \\
\leq\left\|w_{\alpha 1 h}^{n+1}-w_{\alpha 1 h}\right\|_{1}+\left\|w_{\alpha 1 h}-w_{01 h}\right\|_{1}+\left\|w_{01 h}-w_{01}\right\|_{1} \\
\lim \alpha \rightarrow 0,\left\|w_{\alpha 1 h}-w_{01 h}\right\|_{1} \rightarrow 0
\end{gathered}
$$

$$
\begin{aligned}
& \left\|w_{\alpha 1 h}^{n+1}-w_{01}\right\|_{1} \leq\left\|w_{\alpha 1 h}^{n+1}-w_{\alpha 1 h}\right\|_{1}+\left\|w_{01 h}-w_{01}\right\|_{1} \\
& \leq\left\|u_{\alpha 1 h}^{n+1}-\left\langle u_{\alpha 1 h}^{n+1}\right\rangle-u_{\alpha 1 h}+\left\langle u_{\alpha 1 h}\right\rangle\right\|_{1} \\
& \quad+\left\|u_{01 h}-\left\langle u_{01 h}\right\rangle-u_{01}+\left\langle u_{01}\right\rangle\right\|_{1} \\
& \leq\left\|u_{\alpha 1 h}^{n+1}-u_{\alpha 1 h}\right\|_{1}+\operatorname{meas}(\Omega)^{-1}\left\|u_{\alpha 1 h}^{n+1}-u_{\alpha 1 h}\right\|_{1} \\
& \quad+\left\|u_{01 h}-u_{01}\right\|_{1}+\operatorname{meas}(\Omega)^{-1}\left\|u_{01 h}-u_{01}\right\|_{1} \\
& \leq 4 C \cdot \alpha^{-2} h^{2}|\log h|^{4}
\end{aligned}
$$

For: $\alpha=\sqrt{h}$ and $h \rightarrow 0$.

The case $\mathrm{i}=2$ is similar.

$$
\lim _{h \rightarrow 0}\left\|w_{\alpha i h}^{n+1}-w_{0 i}\right\|_{i}=0, i=1,2 \text {. }
$$

2)

$$
\begin{aligned}
& \left|\lambda_{\alpha 1 h}^{n+1}-\lambda_{01}\right| \\
& \leq\left|\lambda_{\alpha 1 h}^{n+1}-\lambda_{\alpha 1}\right|+\left|\lambda_{\alpha 1}-\lambda_{01}\right| \\
& \leq\left|\alpha^{n+1}\left\langle u_{\alpha 1 h}^{n+1}\right\rangle-\alpha\left\langle u_{\alpha 1}\right\rangle\right|+\left|\lambda_{1 \alpha}-\lambda_{01}\right| .
\end{aligned}
$$

Hence $\left|\alpha^{n+1}\right| \leq|\alpha|$ : for $\left.\alpha \in\right] 0,1[$

$$
\begin{aligned}
& \left|\lambda_{\alpha 1 h}^{n+1}-\lambda_{01}\right| \\
& \leq \alpha\left|u_{\alpha 1 h}^{n+1}-u_{\alpha 1}\right|+\left|\lambda_{1 \alpha}-\lambda_{01}\right| \\
& \leq \alpha \cdot C \cdot \alpha^{-2} h^{2}|\log h|^{4}+\left|\lambda_{1 \alpha}-\lambda_{01}\right| \\
& \leq C \cdot \alpha^{-1} h^{2}|\log h|^{4}+\left|\lambda_{1 \alpha}-\lambda_{01}\right|
\end{aligned}
$$

$\lim \alpha \rightarrow 0,\left|\lambda_{1 \alpha}-\lambda_{01}\right| \rightarrow 0$, For: $\alpha=\sqrt{h}$ and $h \rightarrow 0$.

The case $i=2$ is similar.

$$
\lim _{h \rightarrow 0}\left|\lambda_{\alpha i h}^{n+1}-\lambda_{0 i}\right|=0, i=1,2 \text {. }
$$

\section{REFERENCES}

[1] M. Boulbrachène, "On Numerical Analysis of the Ergodic Control Quasi-Variational Inequalities," International Mathematical Forum, No. 42, 2009, pp. 2051-2057.

[2] J. P. Zeng and S. Z. Zhou, "Schwarz Algorithm for the Solution of Variational Inequalities with Nonlinear Source Terms," Applied Mathematics and Computation, Vol. 97, No. 1, 1998, pp. 23-35. doi:10.1016/S0096-3003(97)10129-1

[3] P. L. Lions and B. Perthame, "Quasi-Variational Inequalities and Ergodic Impulse Control," SIAM Journal on Control and Optimization, Vol. 24, No. 4, 1986, pp. 604 615.

[4] A. Bensoussan, "Stochastic Control by Functional Analysis Methods," North-Holland Publishing Company, Amsterdam, 1982.

[5] A. Bensoussan and J. L. Lions, "Impulse Control and Quasi-Variational Inequalities," Gauthiers Villars, Paris, 1984.

[6] P. Cortey-Dumont, "Approximation numérique d'une IQV liée a des problèmes de gestion de stock," RAIRO, Anal. Numer, Vol. 14, 1980, pp. 335-346. 
[7] P. G. Ciarlet and P. A. Raviart, "Maximum Principle and Uniform Convergence for the Finite Element Method," Computer Methods in Applied Mechanics and Engineering, Vol. 2, No. 1, 1973, pp. 17-31.

[8] M. Haiour and S. Boulaaras, "Overlapping Domain Decomposition Methods for Elliptic Quasi-Variational Inequalities Related to Impulse Control Problem with Mixed Boundary Conditions," Proceedings-Mathematical Sciences, Vol. 121, No. 4, 2011, pp. 481-493.

[9] B. Perthame, "Some Remarks on Quasi-Variational Inequalities and the Associated Impulsive Control Problem," Annales de l'I. H. P., Section C, Vol. 2, No. 3, 1985, pp. 237-260.

[10] C. Xiao-Chuan, T. P. Matew and M. Vsakis, "Maximum nom Analysis of Ovelapping Non-Matching Grid Discretisations of Elliptic Equation," SIAM Journal on Numerical Analysis, Vol. 5, 2000, pp. 1709-1728.

[11] J. Bramble, J. Pascial, J. wang and J. xu, "Convergence Estimates for Product Iterative Methods with Applications to Domain Decomposition," Mathematics of Computation, Vol. 57, 1991, pp. 1-21. doi:10.1090/S0025-5718-1991-1090464-8

[12] M. Boulbrachène, P. Cortey-Dumont and J. C. Miellou, "Approximation Convergence for a Subdomain Decomposition Method," 1er Symposium International sur la Méthode de Sous-Domaine, Paris, 1987.
[13] M. Boulbrachène and S. Saadi, "Maximum Norm Analysis of an Overlapping Nonmatching Grids Method for the Obstacle Problem," Hindawi Publishing Corporation, Cairo, 2006, pp. 1-10.

[14] M. Dryja, "An Additive Schwarz Algorithm for Two-and Three-Dimensional Finite Element Elliptic Problems, In: T. Chan, et al., Eds., Domain Decomposition Methods, Philadephia, SIAM, 1989, pp. 168-172.

[15] M. Dryja and O. Widlund, "Some Domain Decomposition Algorithms for Elliptic Problems," In: L. Hayes and D. Kincaid, Eds, Iterative Methods for Large Systems, Academic Press, Boston, 1990, pp. 273-291.

[16] P. Cortey-Dumont, "Sur les inéquations variationnelles à opérateurs non coercifs," M2AN, Vol. 19, 1985, pp. 195212.

[17] P. Cortey-Dumont, "On Finite Element Approximation in the $L^{\infty}$-Norm of Variational Inequalities with Nonlinear Operators," Numerische Mathematik, Vol. 47, No. 1, 1985, pp. 45-57. doi:10.1007/BF01389875

[18] T. Chan, T. Hou and P. Lions, "Geometry Related Convergence Results for Domain Decomposition Algorithms," SIAM Journal on Numerical Analysis, Vol. 28, No. 2, 1991, pp. 378-391. doi:10.1137/0728021 